\title{
Metal-like heat conduction in laser-excited InSb probed by picosecond time-resolved $x$-ray diffraction
}

\author{
P. Sondhauss, ${ }^{1,2}$ O. Synnergren, ${ }^{1}$ T. N. Hansen, ${ }^{1}$ S. E. Canton, ${ }^{3}$ H. Enquist, ${ }^{1}$ A. Srivastava,,${ }^{1}$ and J. Larsson ${ }^{1, *}$ \\ ${ }^{1}$ Department of Physics, Lund University, P.O. Box 118, 22100 Lund, Sweden \\ ${ }^{2}$ MAX-lab, Lund University, P.O. Box 118, 22100 Lund, Sweden \\ ${ }^{3}$ Department of Chemical Physics, Lund University, P.O. Box 118, 22100 Lund, Sweden
}

(Received 23 April 2008; revised manuscript received 12 July 2008; published 10 September 2008)

\begin{abstract}
A semiconductor ( $\mathrm{InSb}$ ) showed transient metal-like heat conduction after excitation of a dense electron-hole plasma via short and intense light pulses. A related ultrafast strain relaxation was detected using picosecond time-resolved x-ray diffraction. The deduced heat conduction was, by a factor of 30, larger than the lattice contribution. The anomalously high heat conduction can be explained once the contribution from the degenerate photocarrier plasma is taken into account. The magnitude of the effect could provide the means for guiding heat in semiconductor nanostructures. In the course of this work, a quantitative model for the carrier dynamics in laser-irradiated semiconductors has been developed, which does not rely on any adjustable parameters or $a d$ hoc assumptions. The model includes various light absorption processes (interband, free carrier, two photon, and dynamical Burstein-Moss shifts), ambipolar diffusion, energy transport (heat and chemical potential), electrothermal effects, Auger recombination, collisional excitation, and scattering (elastic and inelastic). The model accounts for arbitrary degrees of degeneracy.
\end{abstract}

DOI: 10.1103/PhysRevB.78.115202

PACS number(s): 78.47.jc, 61.05.cp, 72.20.Ht, 78.20.Nv

\section{INTRODUCTION}

Metals at room temperature are excellent heat conductors due to the high density and high mobility of the conduction electrons. The phonon contribution to heat conduction is usually much smaller. Counterintuitively, semiconductors do not become better, more metal-like heat conductors as temperature increases despite of increasing free-carrier numbers. This is because the carrier mobility decreases due to scattering from an increasing number of phonons. The case is similar to chemical doping where the effect of increased carrier density is equally counteracted by reduced carrier mobility due to crystal defects introduced by the doping. Laser doping on the other hand, i.e., the excitation of electron-hole pairs by absorption of short laser pulses, does not reduce the carrier mobility since the lattice temperature remains low.

When a short light pulse with photon energies larger than the energy gap interacts with a semiconductor, electrons are excited from the valence into the conduction band mainly via interband absorption. In a few tens of femtoseconds, the electrons thermalize, i.e., reach a Fermi-Dirac distribution, via carrier-carrier collisions. ${ }^{1}$ On a longer time scale (of the order of picoseconds), the carriers thermalize also with the lattice, which is facilitated by inelastic carrier-phonon scattering. Shifting carrier populations as well as the heating of the lattice generate stress via deformation potential and thermal expansion, respectively. The subsequent rapid expansion triggers a strain wave, ${ }^{2}$ which can be described as a distribution of coherent acoustic phonons. As the plasma density drops by ambipolar diffusion and recombination, the nearsurface strain due to the deformation potential relaxes quickly, such that only thermal expansion remains after few tens of picoseconds.

In the present time-resolved x-ray diffraction (TXRD) study, it was found that this relaxation happens on the time scale of a picosecond. This unexpectedly fast relaxation shows that ultrafast carrier and heat transport transfer the laser-deposited energy deeper into the crystal. This transfer has been studied by comparing the experimental observations with simulations based on a sophisticated transport model. ${ }^{3}$ This model takes full account of varying degrees of carrier degeneracy, variation of transport coefficients over time and depth, as well as separate quasi-Fermi levels for electrons and holes. The measurement of the near-surface strain was facilitated by exciting the sample with multiple laser pulses in short succession, ${ }^{4-6}$ a technique that can be used to either enhance or suppress the x-ray diffraction signal from the acoustic strain component. This allowed the clear identification of the contribution from the quickly relaxing near-surface strain.

\section{PREVIOUS WORK}

Over the last decades, laser-solid interaction under comparable conditions has been extensively studied using probes in the visible, near-infrared, or ultraviolet regime, which are mostly susceptible to the carrier density. ${ }^{2,7-9}$ To a smaller extent these probes are also sensitive to strain. ${ }^{2,9}$ Light in the visible, near-infrared, or ultraviolet regime though cannot directly resolve the shape of carrier density profiles in strongly absorbing materials such as semiconductors or metals since it does not penetrate sufficiently deep into the material. Indirectly, this information can be obtained by probing the acoustic strain. This method is not limited by small penetration depths because the acoustic strain propagates through the probed area and, in this way, spatial information is transferred into the time domain. However, the evidence is rather indirect. The laser generated acoustic strain depends on the entire chronology of carrier density and lattice temperature at all depths, i.e., it is both a noninstantaneous and nonlocal function of the parameters that one intends to probe. 

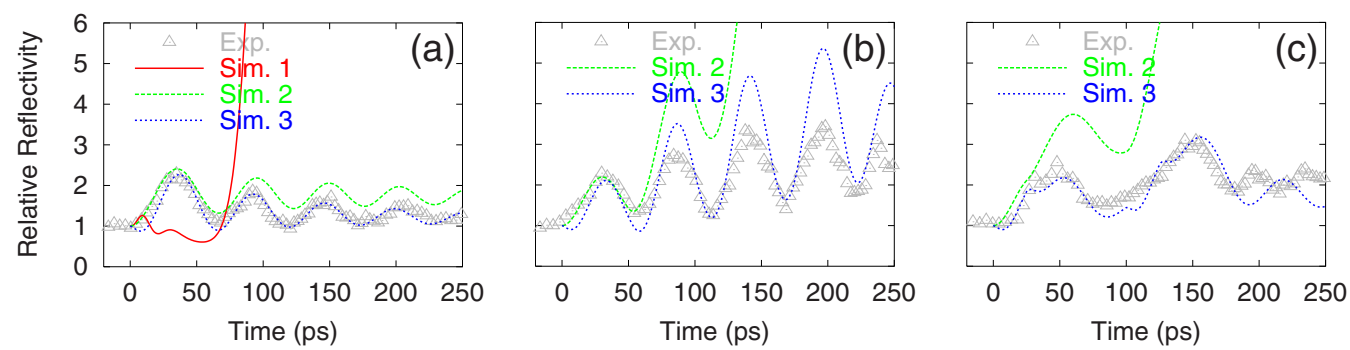

FIG. 1. (Color online) Modulated x-ray reflectivity for three different laser pump schemes: (a) single laser pulse, (b) four equidistant pulses $50 \mathrm{ps}$ apart, and (c) four nonequidistant pulses at $0,25,100$, and $125 \mathrm{ps}$. Measurements were taken at $E=E_{0}-9 \mathrm{eV}$ (triangles) $\left(E_{0}=3630 \mathrm{eV}\right.$, energy at rocking curve peak). The simulations are based on the phenomenological Thomsen model (description in text). "Sim.1" includes both thermal expansion and electronic strain (red solid line), "Sim. 2" includes only thermal expansion (green dashed line), and "Sim. 3" includes only thermal strain with a fast decaying near-surface strain (blue fine dashed line).

X-ray probes on the other hand penetrate deeply and hence can resolve the profile of the nonpropagating nearsurface strain as well. The near-surface strain is a linear and local function of carrier density and lattice temperature. Since carrier dynamics after laser excitation are very complex, we believe that, for reliable conclusions, both strains must be probed as done in the presented work by TXRD.

TXRD studies have given insights into laser-solid interaction. Many of these studies focused on coherent acoustic phonons. ${ }^{10-15}$ In addition, TXRD provided different perspectives on nonthermal melting, ${ }^{16,17}$ optical phonons, ${ }^{18}$ and folded phonon modes in semiconductor heterostructures. ${ }^{19,20}$ A few studies also included phenomenological transport models. ${ }^{21,22}$

\section{EXPERIMENT}

The experiment was performed at beamline D611 at the MAX-lab synchrotron-radiation facility. The setup is described in more detail elsewhere. ${ }^{23}$ The sample was excited using 35 fs laser pulses with a wavelength centered at 790 $\mathrm{nm}$. Prepulses and amplified spontaneous emission were less than $1 \%$ of the main pulse. The laser repetition rate was 4.25 $\mathrm{kHz}$, and the typical acquisition time was $20 \mathrm{~min}$. The laser spot size was $500 \times 1000 \mu \mathrm{m}^{2}$ [full width at half maximum (FWHM)], and the energy per laser pulse was $7 \mu \mathrm{J}$. The $p$-polarized laser pulses were incident at an angle of $54^{\circ}$ to the surface normal, resulting in a reflectivity of $21 \%$. The sample, an InSb crystal cut at $8^{\circ}$ to the (111) plane, was probed by $\mathrm{x}$-ray radiation with an energy around $3630 \mathrm{eV}$ and an effective bandwidth of $\Delta E / E \approx 2 \times 10^{-4}$. The temporally modulated signal from the 111 reflection in Bragg geometry was recorded by a streak camera equipped with a CsI transmission photocathode. ${ }^{24}$ The probed area was 170 $\times 670 \mu \mathrm{m}^{2}$, which is considerably smaller than the laser focal spot size. Under the assumption of a Gaussian beam profile, the laser fluence was about $1.4 \mathrm{~mJ} / \mathrm{cm}^{2}$. This value is well below the melting or damage threshold of InSb.

Three different laser excitation schemes were used: single pulse, four equidistant pulses separated by $50 \mathrm{ps}(0,50,100$, and 150), and four nonequidistant pulses $(0,25,100$, and 125). For all schemes, the laser generated strain was probed by $\mathrm{x}$ rays with the energy $E=E_{0} \pm 9 \mathrm{eV}$, where $E_{0}$ $=3630 \mathrm{eV}$ is the energy right in the center of the InSb 111 reflection peak. The x-ray angle of incidence was kept constant. In this setup, the energy offset defines which phonon wavelength is probed. ${ }^{15,25}$ Under the described conditions, coherent phonons from only one particular dispersion branch are excited efficiently. If the sample surface were cut exactly parallel to the (111) planes, this would be strictly valid and only phonons in the longitudinal-acoustic phonon branch would be excited. Since the (111) planes are actually at an angle of $8^{\circ}$ with respect to the surface, the situation is more complex. ${ }^{26,27}$ First of all, a strict separation between longitudinal and transversal modes cannot be made. All acoustic modes have both longitudinal and transversal components. Since the angle is relatively small, one acoustic branch is still mostly longitudinal, i.e., its longitudinal component is considerably larger than its transversal ones while the other two branches are mostly transversal. Second, coherent phonons in more than one acoustic branch are excited. The solution of the inhomogeneous anisotropic wave equation ${ }^{27}$ under the given conditions shows that also one quasitransversal wave is excited with an amplitude less than $13.5 \%$ of the quasilongitudinal one. Since x-ray diffraction is only sensitive to the strain component in the direction of the reciprocal-lattice vector, ${ }^{25}$ even an equally strong transversal wave would have only $14 \%$ of the effect on $\mathrm{x}$-ray reflectivity compared to a longitudinal one. Hence, the x-ray signal from the quasitransversal wave is altogether less than $2 \%$ of that from the quasilongitudinal one, which is well below the noise level and therefore neglected here. Shear components of the quasilongitudinal mode and longitudinal components of the quasitransversal modes are found to be smaller than $4 \%$ of the dominant components and therefore neglected here as well.

For the single pulse excitation scheme, additional modes at $\Delta E= \pm 7$ and $\pm 5 \mathrm{eV}$ were probed in order to get an idea of the width of the coherent phonon spectrum, and hence the width of the acoustic strain profile.

The temporal modulations of the x-ray reflectivity for the various schemes are shown in Fig. 1. The oscillatory part of the reflectivity, which is clearly visible in the single pulse and in the four equidistant pulse excitation schemes, originates from the acoustic part of the strain. In the case of the four equidistant pulses, the oscillation amplitude of the $\mathrm{x}$-ray reflectivity increases from one period to the next in contrast to the single pulse data. In the case of the nonequidistant pulses, the oscillations are efficiently cancelled after a half 

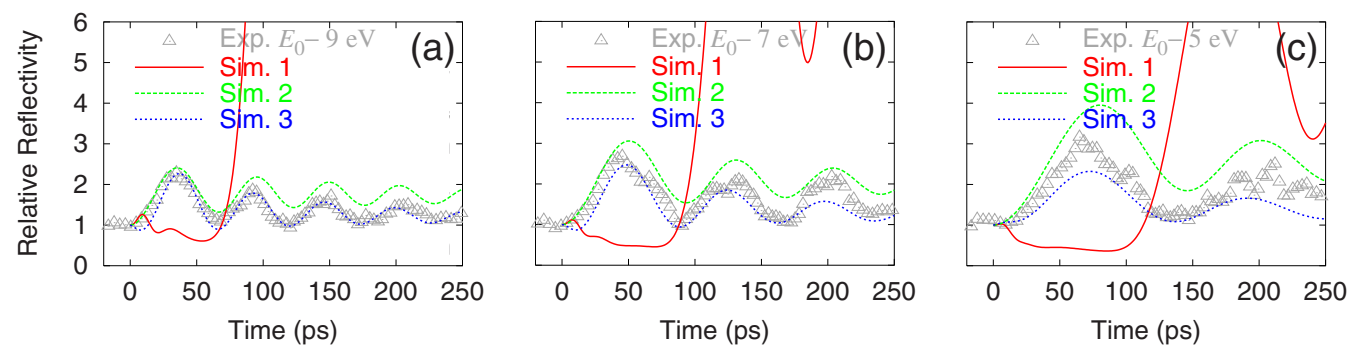

FIG. 2. (Color online) Modulated x-ray reflectivity after excitation with a single laser pulse measured at three different x-ray energy offsets (a) $E-E_{0}=-9$, (b) -7 , and (c) $-5 \mathrm{eV}$. The simulations are again based on the phenomenological Thomsen model (description in text). "Sim.1" includes both thermal expansion and electronic strain (red solid line), "Sim. 2" includes only thermal expansion (green dashed line), and "Sim. 3" includes only thermal strain with a fast decaying near-surface strain (blue fine dashed line).

period. ${ }^{4,6}$ The nonpropagating near-surface strain manifests itself in a nonoscillatory modulation of the x-ray reflectivity. The effects of the acoustic strain and the nonpropagating near-surface strain are generally entangled. For example, a relatively flat near-surface strain profile basically shifts the reflection peak and hence the effective energy offset. This leads to modifications of oscillation period and amplitude. The present study was possible because the signal ratio from both components could be controlled by the different multipulse excitation schemes. To enhance comparability the same number of laser pulses in all multipulse excitation schemes was used, providing identical total fluence and fluence per pulse. More than four pulses in a row did not exhibit significant improvements, mainly due to the fact that the strain profiles from four pulses with 50 ps distance already cover a depth of $700 \mathrm{~nm}$ representing a considerable fraction of the $\mathrm{x}$-ray penetration depth. ${ }^{6}$

\section{PHENOMENOLOGICAL MODEL}

In earlier TXRD studies, ${ }^{10,12,13,15}$ a phenomenological acoustic model for the strain generation in laser-excited solids by Thomsen et al. ${ }^{2}$ provided good agreement with experimental data. However, it did not give insights into the physical processes in the laser-excited material. The Thomsen model incorporates two effects giving rise to strain after laser excitation. First, electronic strain that arises from the bonding or antibonding character of the photoexcited electronic states. It is described by the deformation potential. For a given electron-hole pair density $n$, the uniaxial electronic strain (no expansion perpendicular to the surface normal) in a semiconductor is given by ${ }^{28}$

$$
\eta_{\mathrm{el}}(z)=\frac{1+v}{1-\nu} \frac{1}{3} n(z) \frac{\partial E_{g}}{\partial P},
$$

where $\nu$ stands for the Poisson ratio and $\partial E_{g} / \partial P$ for the variation of the energy gap under hydrostatic pressure.

The second effect is thermal expansion:

$$
\eta_{\mathrm{th}}(z)=\frac{1+\nu}{1-\nu} \beta \Delta T(z),
$$

where $\beta$ stands for the thermal-expansion coefficient and $\Delta T$ for the increase in lattice temperature. The Thomsen model is often used in a way where the laser absorption depth is an adjustable parameter to fit the experimental data. This is motivated by an increased effective absorption depth ${ }^{21}$ due to energy transport following the laser excitation.

The need for a more sophisticated model becomes evident when comparing phenomenologically modified versions of Thomsen's model to our data (Figs. 1 and 2). Thomsen's original model, taking electronic strain into account, strongly deviates from the experimental data from the single laserpulse excitation scheme ["sim. 1" in Fig. 1(a)]. The predicted strain is by about a factor of ten too high. By disregarding electronic strain and using an effective absorption depth of $175 \mathrm{~nm}$ rather than the literature value ${ }^{29}$ of $95 \mathrm{~nm}$ ["sim. 2" in Fig. 1(a)], a fair agreement is obtained. This first modification of Thomsen's model fails strikingly when applied to the multipulse schemes assuming a simple superposition of single pulse strain profiles ["sim. 2" in Figs. 1(b) and 1(c)]. The measured pulse-to-pulse increase in the $\mathrm{x}$-ray diffraction signal is considerably smaller than the linear increase in the model. Therefore, the second modification includes relaxation of near-surface strain via a general diffusion process where the strain itself is assumed to diffuse following Fick's law. A characteristic diffusion time of $\tau=2$ ps gives a much better fit for all three excitation schemes ("sim. 3" in Fig. 1). This corresponds to a huge diffusion constant of $10 \mathrm{~cm}^{2} \mathrm{~s}^{-1}$. This value is purely phenomenological since many effects contribute to the observed strain relaxation, including nondiffusion effects such as Auger recombination. Furthermore, such a rapid relaxation would affect the generation of acoustic strain. The reduction in near-surface strain by relaxation is not taken into account when the acoustic strain is calculated, which might explain why it is still overestimated ["sim. 3" in Fig. 1(b)]. There are also indications that the shape of the acoustic strain profile is not correct either. While the last model reproduces the modulated $\mathrm{x}$-ray reflectivity at $\Delta E=-9 \mathrm{keV}$ rather well ["sim. 3" in Fig. 2(a)] a discrepancy arises at x-ray energy offsets of $\Delta E=-7$ and $-5 \mathrm{keV}$ [Figs. 2(b) and 2(c)] that probe longer phonon wavelengths.

When reviewing the three simulations based on the modified Thomsen model, it is clear that, in spite of the obvious flaws, there is a very fast relaxation of the initial near-surface strain. In order to get reasonable agreement, electronic strain had to be neglected and the phenomenological model gives no motivation for this ad hoc assumption. 


\section{VAN DRIEL'S MODEL}

To gain further insights, a model is required that takes into account the relevant physical processes affecting strain generation and relaxation in a laser-irradiated semiconductor. Ideally the model should have no free parameters and should not be based on any ad hoc assumptions. A model that almost meets these requirements has been developed by Van Driel. $^{3}$

\section{A. Transport equations}

Van Driel's model ${ }^{3}$ is a self-consistent model based on the Boltzmann transport equation in the relaxation-time approximation. In this model the electron-hole pair density $n$, the carrier energy density $u$, and the lattice energy density $u_{L}$ fulfill the respective balance equations:

$$
\begin{gathered}
\frac{\partial n}{d t}+\frac{\partial j^{n}}{\partial z}=\left(\frac{\partial n}{\partial t}\right)_{\text {coll }}+\left(\frac{\partial n}{\partial t}\right)_{\text {laser }}, \\
\frac{\partial u}{d t}+\frac{\partial j^{u}}{\partial z}=\left(\frac{\partial u}{\partial t}\right)_{\mathrm{ep}}+\left(\frac{\partial u}{\partial t}\right)_{\text {laser }}, \\
\frac{\partial u_{L}}{d t}-\frac{\partial}{\partial z}\left(\lambda_{L} \frac{\partial T}{d z}\right)=-\left(\frac{\partial u}{\partial t}\right)_{\mathrm{ep}},
\end{gathered}
$$

with the electron-hole pair current density $j^{n}$, and the carrier energy current density $j^{u}$ defined by

$$
\begin{gathered}
j^{n}=-\frac{\sigma_{a}}{e^{2}} \frac{\partial \mu_{a}}{\partial z}+\frac{\sigma_{a} Q_{a}}{e} \frac{\partial T_{e}}{\partial z}, \\
j^{u}=-\lambda_{a} \frac{\partial T_{e}}{\partial z}+\mu_{a} j^{n}-e \pi_{a} j^{n} .
\end{gathered}
$$

Here, $z$ stands for the depth coordinate, $\mu_{a}=\mu_{c}-\mu_{v}$ for the difference between Fermi levels of electrons and holes, and $T_{e}$ for the carrier temperature, which in general can be different from the lattice temperature $T$.

Equation (3) represents the electron-hole pair balance equation. The first term on the right-hand side (rhs), $(\partial n / \partial t)_{\text {coll }}$, describes collisional excitation as well as recombination of electron-hole pairs via the Auger effect. The second term, $(\partial n / \partial t)_{\text {laser }}$, describes the excitation of electronhole pairs in the laser absorption process. The second term on the left-hand side (lhs) stands for changes of the electronhole pair density due to transport. Carrier transport can be broken down into two parts according to the expression for the electron-hole pair current in Eq. (6). The first term on the rhs describes diffusion while the second term describes thermodiffusion. This split up is not unequivocal. Rather than Fermi levels and temperature, one could consider electronhole pair density and temperature as independent variables. The terms for diffusion (density gradient) and thermodiffusion (temperature gradient) would be different because the Fermi levels depend both on carrier density and temperature. In any case, $\sigma_{a}$ and $Q_{a}$ stand for the ambipolar electrical conductivity and the ambipolar Seebeck coefficient (thermopower), respectively.
Equation (4) represents the energy balance equation for the carriers. The first term on the rhs, $(\partial u / \partial t)_{\mathrm{ep}}$, describes the energy loss due to inelastic phonon scattering while the second term, $(\partial u / \partial t)_{\text {laser }}$, describes the energy gain by laser absorption. The second term on the lhs stands for the gain or loss per unit time via energy transport. Energy transport in the electron-hole plasma can be broken down into three parts according to the three terms for the energy current density on the rhs of Eq. (7). The first term describes heat conduction, and the second and third terms describe the transfer of heat and chemical potential, respectively, associated with the electron-hole pair current. The transport coefficients $\lambda_{a}$ and $\pi_{a}$ stand for the thermal conductivity of the electron-hole plasma and the Peltier coefficient, respectively. The Peltier coefficient describes the amount of heat transported per unit electrical current. For drifting electrons, for example, the Peltier coefficient is always negative and thus the heat flows into the same direction as the particle current. The distinction between heat conduction and heat transport by drifting electron-hole pairs is somewhat artificial here. Heat conduction also involves carrier exchange between warm and cold regions. The only difference to heat transport by an ambipolar carrier flow is that the electric currents cancel out in each individual band and not only in all bands together. Furthermore, according to Eq. (6) the electron-hole pair current is also driven by the temperature gradient. Hence, the heat current associated with an electron-hole pair current has a lot in common with convection in fluids and therefore can be considered as a form of heat conduction.

Equation (5) finally represents the energy balance equation for the lattice. The second term on the lhs describes lattice heat conduction with the lattice thermal conductivity $\lambda_{L}$. The energy balance equation for the lattice is linearly coupled to that for the electron-hole plasma via the inelastic electron phonon-scattering term $(\partial u / \partial t)_{\mathrm{ep}}$.

Three bands are involved in carrier and energy transport: two valence bands (heavy and light holes) and the conduction band. The carrier transport coefficients $\sigma_{a}, Q_{a}, \lambda_{a}$, and $\pi_{a}$ are therefore weighted sums of three single band coefficients. These sums are determined from the conditions for ambipolar flow, $j_{c}+j_{h}+j_{l}=0$, and the condition for thermal equilibrium between heavy and light holes, $\mu_{h}=\mu_{l}$. For example, the electrical conductivities of the two valence bands have to be added to obtain the overall valence-band conductivity, $\sigma_{v}=\sigma_{h}+\sigma_{l}$, whereas the resistivities of valence and conduction bands have to be added to obtain the total ambipolar conductivity, $\sigma_{a}^{-1}=\sigma_{v}^{-1}+\sigma_{c}^{-1}$. Different sum rules apply for other transport coefficients. The rules for two bands in thermal equilibrium, applicable to the two valence bands, are given in standard textbooks. ${ }^{30}$ The rules for an ambipolar flow can be found in Van Driel's work. ${ }^{3}$

The single band transport coefficients are given by band integrals similar to that for the electrical conductivity, ${ }^{30}$

$$
\sigma\left(\mu, T_{e}\right)=-\frac{e^{2}}{3} \int d E \rho(E) \tau_{m}(E) v^{2}(E) \frac{\partial f}{\partial E}\left(E-\mu, T_{e}\right)
$$

where $\rho$ stands for the density of electronic states, $\tau_{m}$ for the momentum relaxation time, $v$ for the carrier group velocity, 
and $f$ for the Fermi-Dirac distribution function. The integral expressions for the other transport coefficients can be found in standard textbooks. ${ }^{30}$ All transport coefficients are in general functions of carrier temperature and Fermi level. Carrier temperature and Fermi level in turn are functions of electronhole pair density and carrier energy density implicitly given by the integral equations

$$
n\left(\mu, T_{e}\right)=\int d E \rho(E) f\left(E-\mu, T_{e}\right),
$$

and

$$
u\left(\mu, T_{e}\right)=\int d E \rho(E) E f\left(E-\mu, T_{e}\right) .
$$

Electron-hole pair density and energy density vary considerably over depth and time. Hence, also temperature and Fermi levels are functions of depth and time, and with it all transport coefficients. The transport coefficients depending on electron-hole and energy density means parametric coupling between Eqs. (3) and (4).

\section{B. Basic assumptions}

A major assumption in Van Driel's model is that of a partial local thermal equilibrium. Only partial because not all thermodynamic subsystems are in equilibrium with respect to both energy and particle exchange. No thermal equilibrium is assumed between the free carriers and the lattice, i.e., $T_{e} \neq T$. Holes and electrons are assumed to be in mutual thermal equilibrium with respect to energy exchange but not with respect to particle exchange, i.e., $\mu_{c} \neq \mu_{v}$. The assumption of a partial local equilibrium allows for the description of the energy spread of electrons and holes by two FermiDirac distribution functions with only three parameters, namely $\mu_{c}, \mu_{v}$, and $T_{e}$. This assumption is justified by the extremely short energy relaxation time due to carrier-carrier scattering. For electron-hole pair densities around $10^{20} \mathrm{~cm}^{-3}$, this is only a few tens of femtoseconds. ${ }^{1}$ Therefore processes occurring on a longer time scale can be safely assumed as quasistatic. There is one effect where this assumption is not entirely justified, namely the dynamical Burstein-Moss shift, also known as band filling. ${ }^{31}$ It is the shift of the interband absorption edge to higher photon energies due to the depletion of electrons in the valance band and/or filling of electronic states in the conduction band. For the occupation probabilities, Fermi-Dirac distribution functions are assumed. The effect takes place on a time scale of the laserpulse duration, which in the presented study is comparable to the carrier-carrier energy relaxation time. Band filling though turns out to have only a small effect at the laser fluence used in the experiment and thus the overall error introduced by assuming thermal distributions is only marginal. Another basic assumption is the validity of the relaxation-time approximation. Since carrier temperatures of several thousand kelvin are reached, all relevant scattering processes, namely polaroptical scattering, and acoustic and optical deformationpotential scatterings, can be considered quasielastic. Therefore a momentum relaxation time can be defined and hence the relaxation-time approximation is valid. ${ }^{32,33}$ Another assumption is implicit in the form of the transport equations given above. All gradients and transport coefficients are scalar. This implies one dimensionality, i.e., one spatial coordinate is sufficient to describe spatial variation. This assumption is justified by the experimental geometry: laser absorption, heat transport, and all other processes happen in a thin surface layer with a thickness of the order of $1 \mu \mathrm{m}$. The lateral dimensions defined by the laser focal spot size, on the other hand, are by about a factor of 100 larger. Ergo, gradients along the surface normal are by a factor of hundred steeper than gradients perpendicular to it and hence: $(\partial / \partial x, \partial / \partial y, \partial / \partial z) \approx(0,0, \partial / \partial z)$.

\section{Extensions}

Van Driel's model has been extended in one major respect: carrier-phonon scattering is treated in more detail. Van Driel assumes the momentum relaxation time to be proportional to $E^{-1 / 2}$, which is the case for deformation-potential scattering. Acoustic deformation-potential scattering is typically the dominant scattering process in nonpolar semiconductor crystals at room temperature. The momentum relaxation time for acoustic deformation-potential scattering is given by ${ }^{32,34}$

$$
\frac{1}{\tau_{m \text { def }}}=\frac{v(E)}{l_{\text {def }}},
$$

with $l_{\mathrm{def}}=\pi \hbar^{4} \rho_{\text {mass }} v_{L}^{2} / m^{2} E_{a}^{2} k_{B} T$ standing for the mean-freepath length, $\rho_{\text {mass }}$ for the crystal's mass density, $v_{L}$ for the speed of the longitudinal-acoustic mode, $m$ for the effective carrier mass, and $E_{a}$ for the acoustic deformation potential. In polar semiconductors such as InSb on the other hand, polar-optical scattering usually predominates with $\tau_{m \text { pol }}$ $\propto E^{+1 / 2}$ or more specifically ${ }^{32,33}$

$$
\frac{1}{\tau_{m \text { pol }}}=\frac{e^{2}}{2 \pi \hbar^{2}}\left(\varepsilon_{\infty}^{-1}-\varepsilon_{s}^{-1}\right) \frac{\hbar \omega_{\mathrm{ph}}}{v(E)}\left[2 N\left(\hbar \omega_{\mathrm{ph}}, T\right)+1\right],
$$

where $N\left(\hbar \omega_{\mathrm{ph}}, T\right)$ stands for the Bose-Einstein distribution function, and $\varepsilon_{\infty / s}$ for the optical and static permittivity, respectively. However, through laser excitation, deformationpotential scattering is enhanced by the increased number of highly energetic carriers with very short deformationpotential scattering times, $\tau_{m \text { def }} \propto E^{-1 / 2}$. Hence, in laserexcited polar semiconductors, deformation-potential scattering must be taken into account as well. The combined momentum relaxation time for both scattering processes, $\tau_{m}^{-1}=\tau_{m \text { def }}^{-1}+\tau_{m \text { pol }}^{-1}$, cannot be expressed by a simple power law $\propto E^{\alpha}$. This would imply high computational costs for the determination of transport coefficients because integrals like that in Eq. (8) cannot be expressed by Fermi integrals. This can be avoided by approximating the momentum relaxation time for deformation-potential scattering by $\tau_{m \text { def }}(E)$ $\approx \tau_{m \text { pol }}(E) l_{\mathrm{def}} / l_{\mathrm{pol}}$, where $l_{\mathrm{pol}}$ stand for the average free path length for polar-optical scattering defined by

$$
l_{\mathrm{pol}}=\int d E \quad \rho v \tau_{m \text { pol }} f / \int d E \rho f .
$$


The accuracy of this approach was checked by comparison to the exact integral expressions for the transport coefficients for a wide range of temperatures and electron-hole pair densities. It turned out to be typically better than $20 \%$. Furthermore, detailed expressions for energy relaxation rates $(\partial E / d t)_{\mathrm{ep}}$ are used rather than a constant energy relaxation time as in Van Driel's work. Using expressions directly from the literature for polar-optical scattering ${ }^{35}$ and optical deformation-potential scattering ${ }^{32}$ gave rise to numerical problems in regions where carrier and lattice temperature are close, $T_{e} \approx T$. These problems arose from the fact that the energy rate $(\partial u / d t)_{\mathrm{ep}}$ derived from these expressions is small at $T_{e}=T$ but not zero. By introducing the correction factors, $\exp \left( \pm \hbar \omega_{\mathrm{ph}} / 2 k_{B} T_{e}\right)$, to the phonon absorption rate (upper sign) and the phonon emission rate (lower sign), respectively, this problem could be solved. These correction factors result directly from the condition $(\partial u / d t)_{\mathrm{ep}}=0$ for equal lattice and carrier temperature. The correction is small, i.e., the correction factors are close to one, for the vast majority of carriers if the average thermal carrier energy $k_{B} T_{e}$ is large compared to the phonon energy $\hbar \omega_{\mathrm{ph}}$. The high-temperature limit of the energy relaxation rate for polar-optical scattering is then given by

$$
\begin{aligned}
\left(\frac{\partial E}{\partial t}\right)_{\mathrm{pol}}= & \frac{e^{2}}{\pi \hbar^{2}}\left(\varepsilon_{\infty}^{-1}-\varepsilon_{s}^{-1}\right) \frac{\left(\hbar \omega_{\mathrm{ph}}\right)^{2}}{v(E)} N\left(\hbar \omega_{\mathrm{ph}}, T\right) \\
& \times \sinh \left[\hbar \omega_{\mathrm{ph}}\left(T_{e}^{-1}-T^{-1}\right) / 2 k_{B}\right] .
\end{aligned}
$$

The hyperbolic sine function is a direct consequence of the introduced correction factors. The equivalent expression for optical deformation-potential scattering is

$$
\begin{aligned}
\left(\frac{\partial E}{\partial t}\right)_{\text {def }}= & \frac{1}{2 \pi \hbar^{2}} \frac{\left(m E_{o}\right)^{2}}{a^{2} \rho_{\text {mass }}} v(E) N\left(\hbar \omega_{\mathrm{ph}}, T\right) \\
& \times \sinh \left[\hbar \omega_{\mathrm{ph}}\left(T_{e}^{-1}-T^{-1}\right) / 2 k_{B}\right],
\end{aligned}
$$

with the lattice constant $a$, and the optical deformation potential $E_{o}{ }^{36}$ The total-energy rate $(\partial u / d t)_{\mathrm{ep}}$ is obtained by integrating the energy relaxation rate $(\partial E / d t)_{\mathrm{ep}}=(\partial E / d t)_{\mathrm{pol}}$ $+(\partial E / d t)_{\mathrm{def}}$ over all (initial) electronic states, $(\partial u / d t)_{\mathrm{ep}}$ $=\int d E \rho(\partial E / d t)_{\mathrm{ep}} f$.

Other changes to Van Driel's model were mandatory due to the particularities of InSb. InSb has a nonparabolic conduction band and a narrow-band gap $(0.18 \mathrm{eV}$ at room temperature). The nonparabolicity leads to an energy dependent effective electron mass, $m_{c}(E)$, and affects among other things electron momentum and density of states. Therefore, detailed band shapes and density of state functions for the conduction band ${ }^{37}$ were used. As a direct consequence of the narrow-band gap, the rates of collisional excitation and $\mathrm{Au}-$ ger recombination scale with the square of the electron-hole pair density rather than to the cube,,$^{38,39}(\partial n / \partial t)_{\text {coll }}=[-1$ $\left.+\exp \left(-\mu_{a} / k_{B} T_{e}\right)\right] A n^{2}$.

\section{Strain generation and x-ray diffraction}

Strain generation and $\mathrm{x}$-ray diffraction were modeled on the basis of the results from Van Driel's extended model. For thermal expansion and electronic strain, the expressions in
Eqs. (1) and (2) were used. Since the InSb crystal structure has no inversion symmetry center, and because different electron and hole mobilities can potentially create strong electrical fields, the converse piezoelectric effect ${ }^{40}$ was considered as well,

$$
\eta_{\mathrm{pi}}(z)=\frac{1+v}{1-\nu} d F
$$

where $d$ stands for the piezoelectric modulus and $F$ for the electrical field. The electrical field can be directly determined from the condition of ambipolar flow, $j_{c}+j_{h}+j_{l}=0$. The acoustic strain that arises from the evolution of thermal, electronic, and piezoelectric strains follows from the solution of the inhomogeneous acoustic wave equation. ${ }^{2}$ Finally, $x$-ray diffraction from the strained crystal was modeled within the dynamical theory of x-ray diffraction. The effects of strain

\begin{tabular}{|c|c|c|}
\hline Description & Value & Unit \\
\hline Laser absorption depth ${ }^{\mathrm{a}}$ at $790 \mathrm{~nm}$ & 95 & $\mathrm{~nm}$ \\
\hline Laser refractive index ${ }^{\mathrm{a}}$ at $790 \mathrm{~nm}$ & 4.568 & \\
\hline Two photon absorption coefficient ${ }^{b}$ & 2.5 & $\mathrm{~cm} \mathrm{MW} \mathrm{MW}^{-1}$ \\
\hline Lattice heat capacity ${ }^{\mathrm{c}}$ & 0.207 & $\mathrm{~J} \mathrm{~g}^{-1} \mathrm{~K}^{-1}$ \\
\hline Lattice thermal conductivity ${ }^{\mathrm{d}}$ at $300 \mathrm{~K}$ & 17.4 & $\mathrm{~W} \mathrm{~m}^{-1} \mathrm{~K}^{-1}$ \\
\hline Linear thermal-expansion coefficient ${ }^{\mathrm{c}}$ & $5.37 \times 10^{-6}$ & $\mathrm{~K}^{-1}$ \\
\hline Piezoelectric modulus $^{\mathrm{c}} d_{14}$ & $2.35 \times 10^{-10}$ & $\mathrm{~cm} \mathrm{~V}^{-1}$ \\
\hline Elastic modulus ${ }^{\mathrm{c}} c_{11}$ & $6.472 \times 10^{10}$ & $\mathrm{~N} \mathrm{~m}{ }^{-2}$ \\
\hline Elastic modulus ${ }^{\mathrm{c}} c_{12}$ & $3.645 \times 10^{10}$ & $\mathrm{~N} \mathrm{~m}^{-2}$ \\
\hline Elastic modulus ${ }^{\mathfrak{c}} c_{44}$ & $3.020 \times 10^{10}$ & $\mathrm{~N} \mathrm{~m}^{-2}$ \\
\hline Poisson ratio ${ }^{\mathrm{e}}$ & 0.24 & \\
\hline Mass density ${ }^{c}$ & 5.8 & $\mathrm{~g} \mathrm{~cm}^{-3}$ \\
\hline Speed of long acoustic mode ${ }^{f}$ & 3860 & $\mathrm{~m} \mathrm{~s}^{-1}$ \\
\hline Energy gap $^{\mathrm{c}}$ & 0.18 & $\mathrm{eV}$ \\
\hline Change of energy gap with pressure ${ }^{c}$ & $16 \times 10^{-6}$ & $\mathrm{eV}$ bar $^{-1}$ \\
\hline Effective mass heavy holes ${ }^{\mathrm{c}}$ & 0.4 & $\mathrm{~m}_{0}$ \\
\hline Effective mass light holes ${ }^{c}$ & 0.016 & $\mathrm{~m}_{0}$ \\
\hline Auger coefficient ${ }^{\mathrm{g}}$ & $1.5 \times 10^{-9}$ & $\mathrm{~cm}^{3} \mathrm{~s}^{-1}$ \\
\hline Optical phonon energy ${ }^{c}$ & 23 & $\mathrm{meV}$ \\
\hline Rel. optical permittivity ${ }^{\mathrm{c}}$ & 15.68 & \\
\hline Rel. static permittivity ${ }^{\mathrm{c}}$ & 18 & \\
\hline Optical deformation-potential holes ${ }^{\mathrm{h}}$ & 40.2 & $\mathrm{eV}$ \\
\hline
\end{tabular}
on diffraction are described by a system of two coupled partial differential equations, also known as Takagi-Taupin equations. ${ }^{41}$ For only depth dependent strain gradients, this

TABLE I. Parameters used in the simulation.

${ }^{\mathrm{a}}$ Reference 29.

${ }^{\mathrm{b}}$ Reference 43 .

${ }^{\mathrm{c}}$ Reference 44 .

${ }^{\mathrm{d}}$ Reference 45.

${ }^{\text {e}}$ Calculated from elastic constants for the given sample geometry (Ref. 46).

${ }^{\mathrm{f}}$ Calculated from elastic constants for the given sample geometry (Ref. 27).

${ }^{g}$ Reference 39.

${ }^{\mathrm{h}}$ Reference 36. 

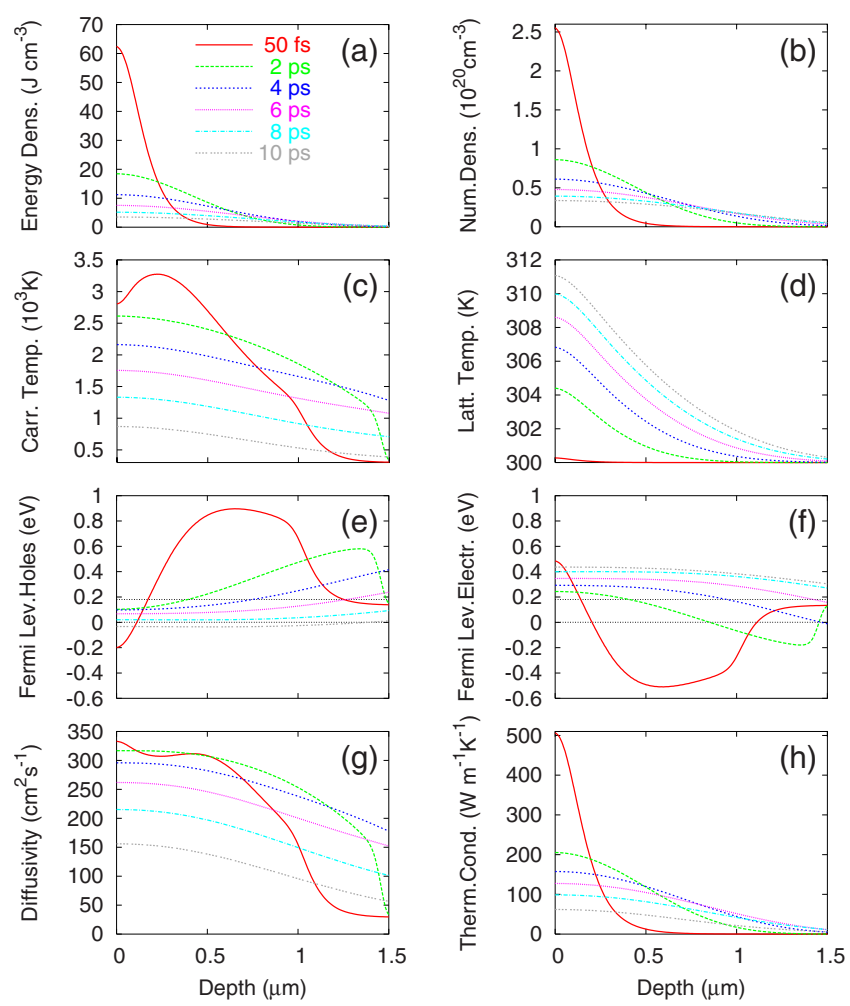

FIG. 3. (Color online) Simulation results at $50 \mathrm{fs}$ (red solid line), 2 ps (green dashed), 4 ps (blue short dashed), 6 ps (magenta dotted), 8 ps (cyan dash dotted), and 10 ps (gray 4-dots) after the peak of a single laser pulse: (a) energy density, (b) electron-hole pair density, (c) carrier temperature, (d) lattice temperature, (e) Fermi-level holes, (f) Fermi-level electrons, (g) ambipolar diffusivity, and (h) ambipolar thermal conductivity. The horizontal lines in the Fermilevel diagrams represent the band edges of valence and conduction band at 0 and $0.18 \mathrm{eV}$, respectively.

system can be reduced to a single ordinary differential equation, ${ }^{42}$ which was solved using a fourth-order variable step size Runge-Kutta algorithm.

\section{SIMULATIONS}

Simulations based on Van Driel's extended model were performed using the parameters given in Table I.

\section{A. Laser absorption and early conditions}

The first result is that under the given conditions the direct photoexcitation of electrons from the valence into the conduction band is the absolute dominant laser absorption process. Other processes such as free carrier or two photon absorption were found to be irrelevant mainly due to the low laser fluence/flux. The dynamical Burstein-Moss shift has only marginal effects, as mentioned earlier.

Initial depth gradients of electron-hole pair and energy density follow closely the exponentially decreasing laserdeposited energy [Figs. 3(a) and 3(b)]. From that the initial carrier temperature and Fermi levels can be deduced [Figs. 3(c), 3(e), and 3(f)] by numerically solving the integral Eqs. (9) and (10). In a surface layer of about $500 \mathrm{~nm}$ thickness, the carrier temperature profile is rather flat and the temperature there amounts to about $3000 \mathrm{~K}$. The flatness arises from the fact that each electron obtains the same amount of energy when photoexcited, and the only parameter that affects the carrier temperature is the ratio between chemical potential and thermal energy. The Fermi levels of electrons and holes on the other hand have extreme values at the surface, and fall/rise quickly with increasing depth [Figs. 3(e) and 3(f)]. At the surface they lie deep within the respective band. At a depth of about $150 \mathrm{~nm}$ they intersect. Beyond that depth the Fermi level of the holes exceeds that of the conduction electrons. The reason for this Fermi-level inversion is the constantly high carrier temperature. Because the carrier numbers decrease quickly, the only way to fulfill Eq. (9) for low carrier densities is to move the Fermi levels far outside the respective band.

\section{B. Auger recombination and collisional excitation}

The region with Fermi-level inversion persists up to a few picoseconds after the laser incidence. The electron-hole plasma there experiences cooling due to collisional excitation. In the region near the surface on the other hand, heating due to Auger recombination prevails. One might expect that this leads to a steeper temperature gradient. This is not the case because at the same time heat is transported away from the surface into larger depths, counteracting Auger heating/ collisional cooling. On the contrary, collisional excitation has a balancing effect on temperature. It prevents low-density plasma regions from heating up to extreme temperatures due to the transfer of high energy electron-hole pairs from denser plasma regions.

\section{Degeneracy and enhanced transport}

Over the full length of a simulation (500 ps), both holes and electrons are found to be degenerate even beyond the relevant depth of $1 \mu \mathrm{m}$. The electrons in the conduction
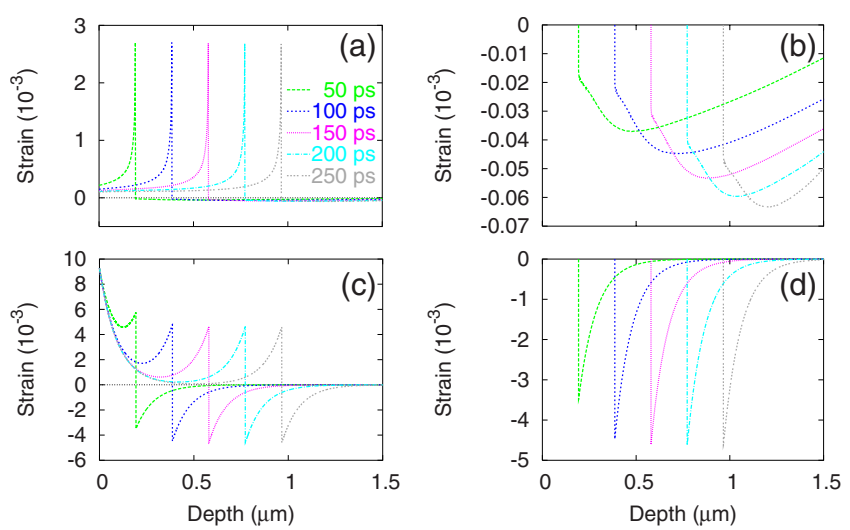

FIG. 4. (Color online) Strain profiles calculated using (a) the Van Driel and (c) the Thomsen models at 50 ps (green dashed line), 100 ps (blue short dashed), 150 ps (magenta dotted), 200 ps (cyan dash dotted), and $250 \mathrm{ps}$ (gray four dot) after incidence of a single laser pulse. To enhance clarity, the compressive strain components according to (b) Van Driel's and (d) Thomsen's models are shown also at a different scale. 

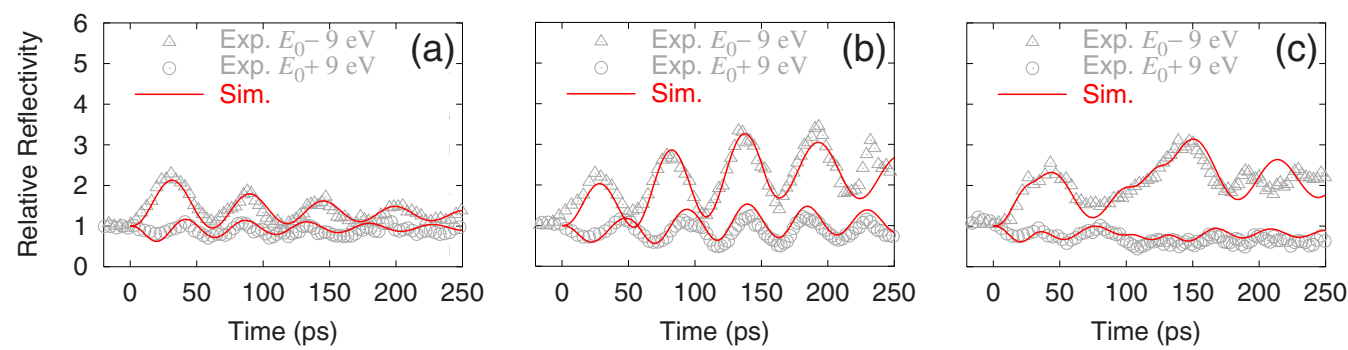

FIG. 5. (Color online) Modulated x-ray reflectivity: pump schemes are the same as in Fig. 1. The simulations are based on Van Driel's extended model including various transport mechanisms and scattering processes (description in text). Measurements were taken at $E=E_{0}$ $-9 \mathrm{eV}$ (triangles) and $E=E_{0}+9 \mathrm{eV}$ (circles).

band are degenerate even before laser excitation because the intrinsic Fermi level lies close to the conduction-band edge. Over depth and time the carriers run through all degrees of degeneracy such that neither the nondegenerate nor the fully degenerate limit can be applied. The high degree of degeneracy in combination with high carrier densities and temperatures enhance transport capacities enormously. Compared to unexcited InSb ambipolar diffusivity increases tenfold and carrier thermal conductivity 10000 fold [Figs. $3(\mathrm{~g})$ and $3(\mathrm{~h})]$. The thermal conductivity of the carrier gas even exceeds that of the lattice by a factor of 30 , making heat conduction basically metal-like. Nevertheless most heat is transported by drifting electron-hole pairs. Diffusion turns out to be the dominant carrier transport mechanism mainly due to the steepness of the electron-hole pair density gradient. Thermodiffusion and other effects driving the ambipolar carrier current such as the carrier self-energy correction $^{3}$ were found to be negligible.

\section{Scattering and relaxation}

Carrier mobility and, as associated with it, carrier transport capabilities are generally limited by scattering processes. First of all, scattering randomizes the carrier momentum in a particular direction (momentum relaxation), and second, carrier excess energy is transferred to the atoms (energy relaxation). In semiconductor crystals at room temperature (lattice), this happens mainly via phonon scattering. Carrier-carrier scattering is strong but does not change the overall carrier momentum because all carrier species drift in the same direction at equal speed. Energy relaxation by carrier-carrier scattering on the other hand is already intrinsically included in the Van Driel model. By assigning a single temperature to the electron-hole plasma, energy relaxation via carrier-carrier scattering is basically assumed instantaneous.

Since the excess kinetic energy of the carriers is high compared to the phonon energy, many carrier-phononscattering events are required for full energy relaxation. As a consequence energy relaxation via carrier-phonon scattering takes much longer than momentum relaxation. The shortest energy relaxation times were found at the highest levels of excitation, i.e., at early times close to the surface, where deformation-potential scattering is strong and adds to polaroptical scattering. However, the energy relaxation time amounts to $7 \mathrm{ps}$ even then. In reality, energy relaxation takes even longer since scattering becomes weaker as the level of excitation drops.

\section{E. Thermal expansion, electronic, piezoelectric, and acoustic strains}

Strain from the converse piezoelectric effect has been found to be entirely negligible. Electrical fields due to different hole and electron mobilities reach $10^{4} \mathrm{~V} / \mathrm{cm}$, resulting in a strain of the order of $10^{-6}$. In laser-excited heterostructures on the other hand, the converse piezoelectric effect has been found to be the dominant strain generating mechanism. ${ }^{47-49}$ One reason for this disparity is that the electrical fields in a heterostructure due to charge separation in chemical-potential gradients are much stronger.

Slow carrier energy relaxation implies that the lattice temperature rises only slowly and with it thermal expansion. Electronic strain on the other hand is instant and therefore dominates the acoustic wave generation. Modeled strain profiles as function of depth and time are shown in Fig. 4. In
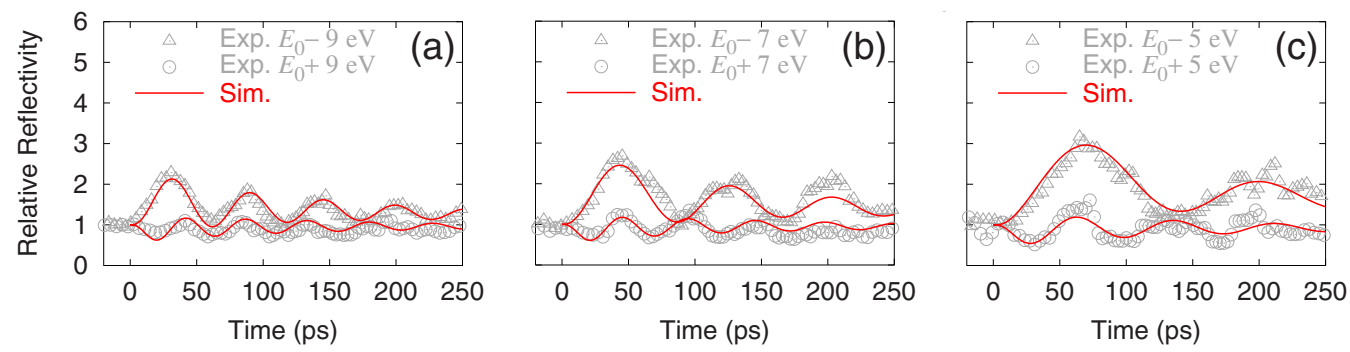

FIG. 6. (Color online) Modulated x-ray reflectivity after excitation with a single laser pulse measured at three different x-ray energy offsets (a) $E-E_{0}= \pm 9$, (b) \pm 7 , and (c) $\pm 5 \mathrm{eV}$. The simulations are based on Van Driel's extended model (description in text). 
turn, the slow increase in thermal strain implies that there is only electronic strain at early times and that the observed ultrafast decay of near-surface strain must be due to a rapid decrease of electron-hole pair density. The simulation shows that this is indeed the case. Due to carrier diffusion and Auger recombination the electron-hole pair density drops by a factor of $1 / e$ within precisely 2 ps. The contribution of carrier transport is larger but nevertheless Auger recombination cannot be neglected. The relaxation of electronic strain happens so quickly that the amplitude of the initially generated acoustic wave decays rapidly. This explains the overestimation of acoustic strain in the Thomsen model where the nearsurface strain remains constant [Figs. 4(c) and 4(d)]. Thermal expansion builds up slower but is more persistent than electronic strain. Already after about 5 ps after laser incidence, thermal expansion exceeds electronic strain. Given the earlier estimated time constant for lattice heat conduction of 600 ps, thermal expansion is basically static on the considered time scale once the carriers have thermalized with the lattice.

\section{CONCLUSIONS}

Given that the extended Van Driel model has no free parameters to fit the experimental data, the agreement between measured and modeled x-ray reflectivity shown in Figs. 5 and 6 is excellent. The model explains the unexpectedly small near-surface strain [Figs. 1(b) and 1(c)], the even smaller amplitude of the acoustic wave [Fig. 1(b)], and reproduces the acoustic wave profile better than the Thomsen model [Figs. 2(b) and 2(c)]. The extended Van Driel model shows that these effects are caused by the ultrafast decay of near-surface strain due to enhanced carrier transport. Associated with this is ultrafast carrier heat conduction.

Carrier heat conduction is so efficient that it is potentially useful for removing heat out of semiconductor nanostructures such as, e.g., integrated electronic circuits, in particular

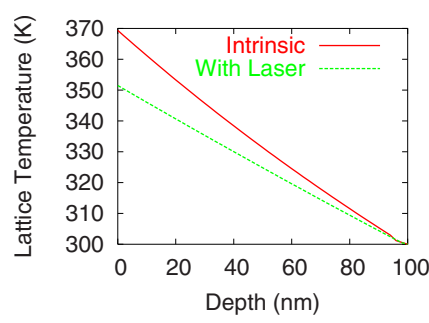

FIG. 7. (Color online) Enhanced heat dissipation by laser pumping: simulated lattice temperature gradient due to a constant heat flow with and without laser excitation.

since the laser does not introduce much heat into the system. In case of the single pulse excitation scheme, the lattice temperature only rises by less than $13 \mathrm{~K}$. More simulations were done to explore this aspect. A heat source was assumed to continuously generate $1 \mathrm{MW} \mathrm{cm}^{-2}$, leading to a temperature difference of $70 \mathrm{~K}$ as this heat dissipates through a $100 \mathrm{~nm}$ thin slab of InSb. Laser exciting the InSb slab reduces this temperature difference by $20 \mathrm{~K}$ (Fig. 7). However, this process is not ultrafast. Due to the large lattice heat capacity and the relatively weak coupling of the carriers with the lattice, it takes about 400 ps over which laser excitation has to be maintained. Using a $3 \mu \mathrm{m}$ wavelength infrared laser, the overall energy input still needs not to be larger than $5 \mathrm{~mJ} \mathrm{~cm}^{-2}$.

\section{ACKNOWLEDGMENTS}

The authors acknowledge Heiner Linke, Lars Samuelson, and Justin Wark for helpful discussions. The authors would like to thank the Swedish Research Council (VR), Knut och Alice Wallenbergs Stiftelse (KAW), the Swedish Foundation for Strategic Research (SSF), the Crafoord Foundation, Carl Tryggers Stiftelse, and the European Commission through the Human Potential Programme. *jorgen.larsson@fysik.lth.se

${ }^{1}$ E. J. Yoffa, Phys. Rev. B 21, 2415 (1980).

${ }^{2}$ C. Thomsen, H. T. Grahn, H. J. Maris, and J. Tauc, Phys. Rev. B 34, 4129 (1986).

${ }^{3}$ H. M. van Driel, Phys. Rev. B 35, 8166 (1987).

${ }^{4}$ A. M. Lindenberg, I. Kang, S. L. Johnson, R. W. Falcone, P. A. Heimann, Z. Chang, R. W. Lee, and J. S. Wark, Opt. Lett. 27, 869 (2002).

${ }^{5}$ É. D. Murray, D. M. Fritz, J. K. Wahlstrand, S. Fahy, and D. A. Reis, Phys. Rev. B 72, 060301(R) (2005).

${ }^{6}$ O. Synnergren, T. N. Hansen, S. Canton, H. Enquist, P. Sondhauss, A. Srivastava, and J. Larsson, Appl. Phys. Lett. 90, 171929 (2007).

${ }^{7}$ D. H. Auston and C. V. Shank, Phys. Rev. Lett. 32, 1120 (1974).

${ }^{8}$ O. B. Wright, U. Zammit, M. Marinelli, and V. E. Gusev, Appl. Phys. Lett. 69, 553 (1996).

${ }^{9}$ O. B. Wright, B. Perrin, O. Matsuda, and V. E. Gusev, Phys. Rev. B 64, 081202(R) (2001).

${ }^{10}$ C. Rose-Petruck, R. Jimenez, T. Guo, A. Cavalleri, C. W. Siders,
F. Ráski, J. A. Squier, B. C. Walker, K. R. Wilson, and C. P. J. Barty, Nature (London) 398, 310 (1999).

${ }^{11}$ A. H. Chin, R. W. Schoenlein, T. E. Glover, P. Balling, W. P. Leemans, and C. V. Shank, Phys. Rev. Lett. 83, 336 (1999).

${ }^{12}$ A. M. Lindenberg, I. Kang, S. L. Johnson, T. Missalla, P. A. Heimann, Z. Chang, J. Larsson, P. H. Bucksbaum, H. C. Kapteyn, H. A. Padmore, R. W. Lee, J. S. Wark, and R. W. Falcone, Phys. Rev. Lett. 84, 111 (2000).

${ }^{13}$ D. A. Reis, M. F. DeCamp, P. H. Bucksbaum, R. Clarke, E. Dufresne, M. Hertlein, R. Merlin, R. Falcone, H. Kapteyn, M. M. Murnane, J. Larsson, Th. Missalla, and J. S. Wark, Phys. Rev. Lett. 86, 3072 (2001).

${ }^{14}$ M. F. DeCamp, D. A. Reis, P. H. Bucksbaum, B. Adams, J. M. Caraher, R. Clarke, C. W. S. Conover, E. M. Dufresne, R. Merlin, V. Stoica, and J. K. Wahlstrand, Nature (London) 413, 825 (2001).

${ }^{15}$ J. Larsson, A. Allen, P. H. Bucksbaum, R. W. Falcone, A. Lindenberg, G. Naylor, T. Missalla, D. A. Reis, K. Scheidt, A. Sjögren, P. Sondhauss, M. Wulff, and J. S. Wark, Appl. Phys. A: 
Mater. Sci. Process. 75, 467 (2002).

${ }^{16}$ A. Rousse, C. Rischel, S. Fourmaux, I. Uschmann, S. Sebban, G. Grillon, Ph. Balcou, E. Förster, J. P. Geindre, P. Audebert, J. C. Gauthier, and D. Hulin, Nature (London) 410, 65 (2001).

${ }^{17}$ A. M. Lindenberg, J. Larsson, K. Sokolowski-Tinten, K. J. Gaffney, C. Blome, O. Synnergren, J. Sheppard, C. Caleman, A. G. MacPhee, D. Weinstein, D. P. Lowney, T. K. Allison, T. Matthews, R. W. Falcone, A. L. Cavalieri, D. M. Fritz, S. H. Lee, P. H. Bucksbaum, D. A. Reis, J. Rudati, P. H. Fuoss, C. C. Kao, D. P. Siddons, R. Pahl, J. Als-Nielsen, S. Duesterer, R. Ischebeck, H. Schlarb, H. Schulte-Schrepping, Th. Tschentscher, J. Schneider, D. von der Linde, O. Hignette, F. Sette, H. N. Chapman, R. W. Lee, T. N. Hansen, S. Techert, J. S. Wark, M. Bergh, G. Huldt, D. van der Spoel, N. Timneanu, J. Hajdu, R. A. Akre, E. Bong, P. Krejcik, J. Arthur, S. Brennan, K. Luening, and J. B. Hastings, Science 308, 392 (2005).

${ }^{18}$ K. Sokolowski-Tinten, C. Blome, J. Blums, A. Cavalleri, C. Dietrich, A. Tarasevitch, I. Uschmann, E. Förster, M. Kammler, M. Horn-von-Hoegen, and D. von der Linde, Nature (London) 422, 287 (2003).

${ }^{19}$ M. Bargheer, N. Zhavoronkov, Y. Gritsai, J. C. Woo, D. S. Kim, M. Woerner, and T. Elsaesser, Science 306, 1771 (2004).

${ }^{20}$ P. Sondhauss, J. Larsson, M. Harbst, G. A. Naylor, A. Plech, K. Scheidt, O. Synnergren, M. Wulff, and J. S. Wark, Phys. Rev. Lett. 94, 125509 (2005).

${ }^{21}$ A. Cavalleri, C. W. Siders, C. Rose-Petruck, R. Jimenez, Cs. Tóth, J. A. Squier, C. P. J. Barty, K. R. Wilson, K. SokolowskiTinten, M. Horn von Hoegen, and D. von der Linde, Phys. Rev. B 63, 193306 (2001).

${ }^{22}$ M. F. DeCamp, D. A. Reis, A. Cavalieri, P. H. Bucksbaum, R. Clarke, R. Merlin, E. M. Dufresne, D. A. Arms, A. M. Lindenberg, A. G. MacPhee, Z. Chang, B. Lings, J. S. Wark, and S. Fahy, Phys. Rev. Lett. 91, 165502 (2003).

${ }^{23}$ M. Harbst, T. N. Hansen, C. Caleman, W. K. Fullagar, P. Jönsson, P. Sondhauss, O. Synnergren, and J. Larsson, Appl. Phys. A: Mater. Sci. Process. 81, 893 (2005).

${ }^{24}$ J. Larsson, Z. Chang, E. Judd, P. J. Schuck, R. W. Falcone, P. A. Heimann, H. A. Padmore, H. C. Kapteyn, P. H. Bucksbaum, M. M. Murnane, R. W. Lee, A. Machacek, J. S. Wark, X. Liu, and B. Shan, Opt. Lett. 22, 1012 (1997).

${ }^{25}$ P. Sondhauss and J. S. Wark, Acta Crystallogr., Sect. A: Found. Crystallogr. 59, 7 (2003).

${ }^{26}$ O. Matsuda, O. B. Wright, D. H. Hurley, V. E. Gusev, and K.
Shimizu, Phys. Rev. Lett. 93, 095501 (2004).

${ }^{27}$ T. Pezeril, V. Gusev, D. Mounier, N. Chigarev, and P. Ruello, J. Phys. D 38, 1421 (2005).

${ }^{28}$ W. B. Gauster, Phys. Rev. 187, 1035 (1969).

${ }^{29}$ D. E. Aspnes and A. A. Studna, Phys. Rev. B 27, 985 (1983).

${ }^{30}$ J. R. Drabble and H. J. Goldsmid, Thermal Conduction in Semiconductors, International Series of Monographs on Semiconductors Vol. 4 (Pergamon, New York, 1961).

${ }^{31}$ P. Lavallard, R. Bichard, and C. Benoît à la Guillaume, Phys. Rev. B 16, 2804 (1977).

${ }^{32}$ K. Seeger, Semiconductor Physics, Springer Series in Solid-State Sciences Vol. 40 (Springer-Verlag, Berlin, 1997).

${ }^{33}$ D. J. Howarth and E. H. Sondheimer, Proc. R. Soc. London 219, 53 (1953)

${ }^{34}$ J. Bardeen and W. Shockley, Phys. Rev. 80, 72 (1950).

${ }^{35}$ E. M. Conwell, Phys. Rev. 143, 657 (1966).

${ }^{36}$ W. Pötz and P. Vogl, Phys. Rev. B 24, 2025 (1981).

${ }^{37}$ J. R. Chelikowsky and M. L. Cohen, Phys. Rev. B 14, 556 (1976); 30, 4828 (1984).

${ }^{38}$ A. Haug, Solid-State Electron. 21, 1281 (1978).

${ }^{39}$ P. M. Fauchet, Phys. Status Solidi B 110, K11 (1982).

${ }^{40}$ J. F. Nye, Physical Properties of Crystals, their Representation by Tensors and Matrices (Clarendon, Oxford, 1967).

${ }^{41}$ S. Takagi, J. Phys. Soc. Jpn. 26, 1239 (1969).

${ }^{42}$ J. Gronkowski, Phys. Rep. 206, 1 (1991).

${ }^{43}$ M. Sheik-bahaei, P. Mukherjee, and H. S. Kwok, J. Opt. Soc. Am. B 3, 379 (1986).

${ }^{44}$ D. Bimberg, R. Blachnik, M. Cardona, P. J. Dean, Th. Grave, G. Harbeke, K. Hübner, U. Kaufmann, W. Kress, O. Madelung, W. von Münch, U. Rössler, J. Schneider, M. Schulz, and M. S. Skolnick, Physics of Group IV Elements and III-V Compounds, Landolt-Börnstein, New Series, Group III Vol. 17 (SpringerVerlag, Berlin, 1982).

${ }^{45}$ G. Busch and E. Steigmeier, Helv. Phys. Acta 34, 1 (1961).

${ }^{46}$ A. Ballato, IEEE Trans. Ultrason. Ferroelectr. Freq. Control 43, 56 (1996)

${ }^{47}$ C. K. Sun, J. C. Liang, and X. Y. Yu, Phys. Rev. Lett. 84, 179 (2000).

${ }^{48}$ G. D. Sanders and C. J. Stanton, Phys. Rev. B 74, 205303 (2006).

${ }^{49}$ Y. C. Wen, L. C. Chou, H. H. Lin, K. H. Lin, T. F. Kao, and C. K. Sun, J. Appl. Phys. 100, 103516 (2006). 